\title{
New Results in Radiative Electroweak Penguin Decays at Babar
}

\author{
Kevin Flood*i \\ California Institute of Technology, Pasadena CA USA 91125 \\ E-mail: kfloodeslac.stanford.edu
}

We report the result of a search for the rare decay $B^{0} \rightarrow \gamma \gamma$ using $426 \mathrm{fb}^{-1}$ of data, corresponding to 226 million $B^{0} \bar{B}^{0}$ pairs, collected on the $\Upsilon(4 S)$ resonance at the PEP-II asymmetric-energy $e^{+} e^{-}$collider using the BABAR detector. We use a maximum likelihood fit to extract the signal yield and observe $21_{-12}^{+13}$ signal events with a statistical signficance of $1.8 \sigma$. This corresponds to a branching fraction $\mathscr{B}\left(B^{0} \rightarrow \gamma \gamma\right)=(1.7 \pm 1.1$ (stat.) \pm 0.2 (syst.) $) \times 10^{-7}$. Based on this result, we set a $90 \%$ confidence level upper limit of $\mathscr{B}\left(B^{0} \rightarrow \gamma \gamma\right)<3.2 \times 10^{-7}$. Using the same dataset, we also set the first upper limit on the rate of the radiative electroweak penguin decay $B^{+} \rightarrow K^{+} \tau^{+} \tau^{-}$.

35th International Conference of High Energy Physics - ICHEP2010,

July 22-28, 2010

Paris France

\footnotetext{
${ }^{*}$ Speaker.

${ }^{\dagger}$ Representing the Babar Collaboration
} 


\section{1. $B^{0} \rightarrow \gamma \gamma$}

In the Standard Model (SM), the decay $B^{0} \rightarrow \gamma \gamma$ occurs through a flavor-changing neutral current (FCNC) transition involving electroweak loop diagrams, as shown in Figure 1. The decays $B^{0} \rightarrow \gamma \gamma$ and $B_{s} \rightarrow \gamma \gamma$ are closely related, with the $b \rightarrow d \gamma \gamma$ transition being suppressed with respect to $b \rightarrow s \gamma \gamma$ by Cabbibo-Kobayashi-Maskawa (CKM) factors $\left(\left|V_{t d}\right|^{2} /\left|V_{t s}\right|^{2} \sim 0.04\right)$. Hadron dynamics introduces uncertainties into the prediction of branching fractions for these decays and may modify the ratio away from the CKM-implied value. While $B^{0} \rightarrow \gamma \gamma$ is expected to have a smaller branching fraction than $B_{s} \rightarrow \gamma \gamma$, a search for the latter faces the experimental challenge of obtaining a large sample of $B_{s}$ mesons, whereas large samples of $B^{0}$ mesons are readily available from $B$ Factory experiments running on the $\Upsilon(4 S)$ resonance.

A leading order calculation for the branching fraction of $B^{0} \rightarrow \gamma \gamma$ [1] yields an estimate of $\left(3.1_{-1.6}^{+6.4}\right) \times 10^{-8}$. This mode is sensitive to new physics that could lead to an enhancement of the branching fraction due to possible contributions of non-SM heavy particles occurring in the loop of the leading-order Feynman diagrams. Such enhancements to the branching fraction for $B^{0} \rightarrow \gamma \gamma$ are less constrained than those for $B_{s} \rightarrow \gamma \gamma$ due to the fact that the $b \rightarrow s \gamma$ transition, responsible for $B_{s} \rightarrow \gamma \gamma$, is known much more accurately than $b \rightarrow d \gamma$. For example, some new physics scenarios involving an extended Higgs sector may considerably enhance the branching fractions with respect to the SM expectation [2]. Supersymmetry with broken $R$-parity [3] also provides scenarios where order of magnitude enhancements are possible. In addition, since the two-photon final state can be either $C P$-even or $C P$-odd, studies of $C P$-violating effects may ultimately be possible.

The best previous upper limit on the branching fraction at $90 \%$ confidence level (CL) is $\mathscr{B}\left(B^{0} \rightarrow \gamma \gamma\right)<6.2 \times 10^{-7}$ set by the Belle experiment [4] using a dataset recorded at the $\Upsilon(4 S)$ resonance with an integrated luminosity of $104 \mathrm{fb}^{-1}$. For the related process $B_{s} \rightarrow \gamma \gamma$, Belle has set a an upper limit on the branching fraction of $8.7 \times 10^{-6}(90 \% \mathrm{CL})$ based on $23.6 \mathrm{fb}^{-1}$ of data taken on the $\Upsilon(5 S)$ resonance [5].

We report results from a search for the decay $B^{0} \rightarrow \gamma \gamma$ using a data sample with integrated luminosity of $426 \mathrm{fb}^{-1}$ collected with the BABAR detector [6] at the $\Upsilon(4 S)$ resonance, corresponding to $\sim 226$ million $B^{0} \bar{B}^{0}$ pairs. The analysis does not distinguish between $B^{0}$ and $\bar{B}^{0}$, and hereinafter charge conjugation is implied unless otherwise noted. The analysis is performed "blind" and uses an unbinned extended maximum likelihood fit to extract the signal yield.

We select events that contain at least two photon candidates with energies of $1.15 \leq E_{\gamma}^{*} \leq$
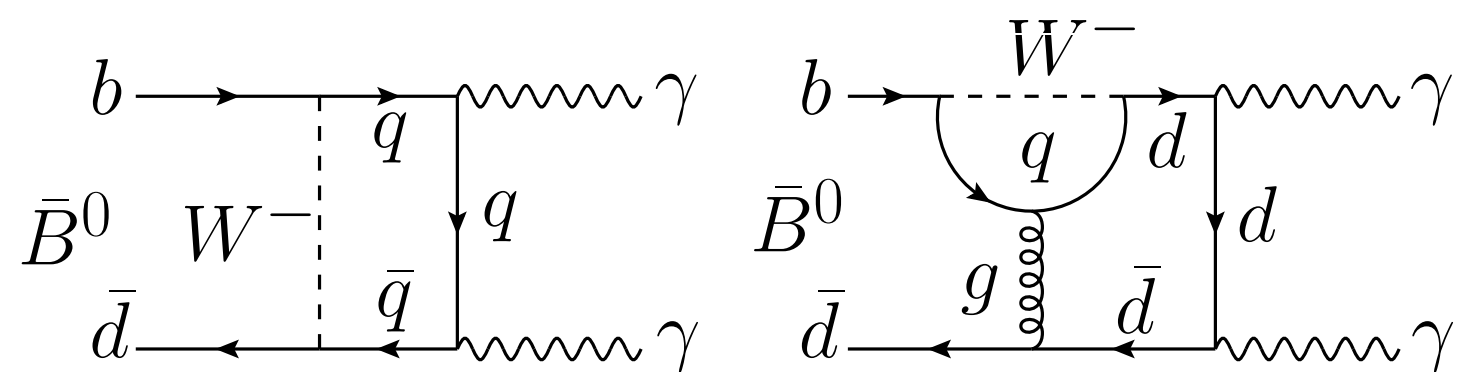

Figure 1: Examples of lowest order SM Feynman diagrams for $B^{0} \rightarrow \gamma \gamma$. The symbol $q$ represents a $u, c$, or $t$ quark. In some new physics scenarios, the $W$-boson may be replaced by a charged Higgs particle. 
$3.50 \mathrm{GeV}$, where the asterisk indicates a quantity in the $\Upsilon(4 S) \mathrm{CM}$ frame, and impose further selections based on isolation and cluster geometry in the electromagnetic calorimeter. We further require the reconstruction time of each $B$ candidate photon to be consistent with the trigger event time. We consider all pair-wise combinations of selected photon candidates in an event and add their fourmomentum to create $B$ meson candidates. The distribution of correctly reconstructed signal $B$ candidates will peak in two weakly correlated variables, $m_{\mathrm{ES}}$ and $\Delta E$. The beam energy substituted mass is defined as $m_{\mathrm{ES}} \equiv \sqrt{E_{\text {beam }}^{* 2}-c^{2} \vec{p}_{\mathrm{B}}^{* 2}} / c^{2}$, while the energy difference is $\Delta E \equiv E_{\mathrm{B}}^{*}-E_{\text {beam }}^{*}$, where $E_{\text {beam }}^{*}$ is the beam energy, and $\vec{p}_{\mathrm{B}}^{*}$ and $E_{\mathrm{B}}^{*}$ are the three-momentum and energy of the $B$ candidate, respectively. For $B^{0} \rightarrow \gamma \gamma$ events, the $m_{\mathrm{ES}}$ distribution will peak at the $B$ meson mass, $5.279 \mathrm{GeV} / c^{2}$ [坷] with a full-width at half maximum (FWHM) of $\sim 6.5 \mathrm{MeV} / c^{2}$. The $\Delta E$ distribution is asymmetric, peaking near zero but with a tail on the negative $\Delta E$ side due to photon energy loss, with a FWHM $\sim 150 \mathrm{MeV}$. We select an event for further analysis if it contains exactly one $B$ candidate with $m_{\mathrm{ES}}>5.1 \mathrm{GeV} / c^{2}$ and $-0.50 \leq \Delta E \leq 0.50 \mathrm{GeV}$. Using simulated signal events, we expect only $\sim 0.06 \%$ of such events to have more than one $B$ candidate. We suppress backgrounds from $e^{+} e^{-} \rightarrow q \bar{q}$ and $\tau^{+} \tau^{-}$processes by requiring the ratio of the second to zeroth Fox-Wolfram moments [8], calculated from the momenta of all charged and neutral particles in an event, to be less than 0.90 . We additionally require that the number of reconstructed charged tracks in an event be greater than two in order to further reject backgrounds from $e^{+} e^{-} \rightarrow \tau^{+} \tau^{-}$events.

The dominant source of backgrounds are photons produced from high-energy $\pi^{0}$ and $\eta$ decays in continuum events. These events are suppressed using a likelihood ratio rejection technique, wherein each $B$ candidate photon is separately combined with all other photons in the event and the invariant mass, $m_{\gamma \gamma^{\prime}}$, and energy of the other photon, $E_{\gamma^{\prime}}$, are used to calculate a likelihood ratio given by

$$
L_{i}=\frac{P_{i}\left(m_{\gamma \gamma^{\prime}}, E_{\gamma^{\prime}}\right)}{P_{\mathrm{sig}}\left(m_{\gamma \gamma^{\prime}}, E_{\gamma^{\prime}}\right)+P_{i}\left(m_{\gamma \gamma^{\prime}}, E_{\gamma^{\prime}}\right)} .
$$

In this equation, $i$ is a label for $\pi^{0}$ or $\eta$, and $P$ represents a two-dimensional probability density function (PDF). For each $B$ candidate photon the pairing that gives the largest value of the likelihood ratio is assigned. A likelihood ratio near $1.0(0.0)$ is consistent with the $B$ candidate photon originating from a $\pi^{0}$ or $\eta$ ( $($ ignal $B$ ). Events where both $B$ candidate photon likelihood ratios are less than 0.84 are selected. For high-energy $\pi^{0}$ decays with $E_{\pi^{0}} \gtrsim 2 \mathrm{GeV}$, the daughter photons may not be separated enough in the EMC to be resolved individually, and the photon clusters are said to be "merged". A merged $\pi^{0}$ can mimic a $B$ candidate photon because the cluster will have the full energy of the parent $\pi^{0}$ and no associated track. We suppress this background using a likelihood based on merged $\pi^{0}$ cluster geometry, selecing only photon candidates unlikely to have arisen from high-energy $\pi^{0}$ decays.

Continuum $q \bar{q}$ events remaining after the $\pi^{0}$ and $\eta$ veto are further rejected with a neural network (NN) utilizing 19 event-shape parameters which exploit differences in event geometry between $b \bar{b}$ and continuum events. We train the NN and validate its performance using simulated $B^{0} \rightarrow \gamma \gamma$ and continuum events. The final selection criteria are optimized using simulated $B^{0} \rightarrow \gamma \gamma$ events and data events in $\Delta E$ and $m_{\mathrm{ES}}$ sidebands, and result in a signal reconstruction efficiency of $\sim 27 \%$ while rejecting $\sim 99.9 \%$ of background events. Backgrounds from $12 B$ decay modes that can peak in the $m_{\mathrm{ES}}$ and $\Delta E$ signal region were studied using large samples of simulated events 
and, using the most recent branching fraction results [7], it is estimated that these modes contribute a total of $1.18 \pm 0.22$ events to the signal region, compared to an expectation of about four signal events predicted from the SM branching fraction.

The signal yield is extracted using a two-dimensional unbinned extended maximum likelihood (ML) fit in the region $m_{\mathrm{ES}}>5.2 \mathrm{GeV} / c^{2}$ and $-0.5 \leq \Delta E \leq 0.5 \mathrm{GeV}$. The likelihood function for a sample of $N$ events with signal, continuum, and peaking $B \bar{B}$ background components is given by

$$
\mathscr{L}=\exp \left(-\sum_{i=1}^{3} n_{i}\right)\left[\prod_{j=1}^{N}\left(\sum_{i=1}^{3} n_{i} \mathscr{P}_{i}\left(\vec{x}_{j} ; \vec{\alpha}_{i}\right)\right)\right],
$$

where $i$ in this equation is an index for the three components in the fit and $n_{i}$ is the event yield for each. Since the correlations between $m_{\mathrm{ES}}$ and $\Delta E$ are found to be small, the signal and continuum background PDFs, $\mathscr{P}_{i}$, are each defined as a product of one-dimensional PDFs in the observables $x_{j} \in\left\{m_{\mathrm{ES}}, \Delta E\right\}$, with parameters $\vec{\alpha}_{i}$. A two-dimensional histogram PDF is used for the peaking background component.

The signal PDF shapes for $m_{\mathrm{ES}}$ and $\Delta E$ are determined from simulated $B^{0} \rightarrow \gamma \gamma$ events. The $m_{\mathrm{ES}}$ distribution is parameterized by a Crystal Ball function [9], and the $\Delta E$ shape is parameterized by a double-sided Gaussian modified to allow for an additional low-side tail. In the ML fit, the signal PDF parameters are fixed to the MC-determined values. All fixed signal parameters are later varied to evaluate the systematic uncertainty that this choice of parameterization has on the signal yield.

The continuum background $m_{\mathrm{ES}}$ distribution is parameterized by an ARGUS shape [10], while the $\Delta E$ distribution is fit with a first-order polynomial. The endpoint of the ARGUS function is fixed to the kinematic limit for $B$ decays $\left(5.290 \mathrm{GeV} / c^{2}\right)$, while all other parameters are allowed to float. The PDF for the peaking background component is parameterized using large samples of simulated exclusive $B$ decays in the form of a two-dimensional histogram PDF in $m_{\mathrm{ES}}$ and $\Delta E$. Both the shape and yield of this component are fixed in the ML fit. The yield is fixed to $3.13 \pm 0.54$ events, which is the predicted number in the fit region determined from the exclusive MC studies. The fixed peaking background PDF shape and yield are later varied to evaluate the systematic uncertainty on the signal yield.

The fit is validated on an ensemble of prototype datasets whose signal and background content and PDF shapes are as expected in the on-resonance data. For the signal content, the datasets are populated with signal events assuming branching fractions of $(1,5,10) \times 10^{-8}$ corresponding to signal yields of 1,6 , and 12 events, respectively. We find no evidence of any significant bias in our fitting procedure using these assumptions.

The on-resonance $\Upsilon(4 S)$ data contains 1679 events after the optimized event selection criteria are applied. We perform the ML fit to extract the signal yield and find $N_{\text {sig }}=21.3_{-11.8}^{+12.8}$ events corresponding to a statistical significance of $1.8 \sigma$. The significance is computed as $\sqrt{2 \cdot \Delta \ln \mathscr{L}}$, where $\Delta \ln \mathscr{L}$ is the difference in the log-likelihood between the best fit to on-resonance data and a fit where the signal yield is fixed to zero. Figure 2 shows projections of the PDF components from the ML fit. For the $m_{\mathrm{ES}}$ projection, the range of $\Delta E$ has been restricted to $-0.30 \leq \Delta E \leq 0.13 \mathrm{GeV}$. For the $\Delta E$ projection, the range of $m_{\mathrm{ES}}$ is restricted to $m_{\mathrm{ES}}>5.27 \mathrm{GeV} / c^{2}$.

Since the signal yield extracted from the ML fit is outside the range initially tested during the fit validation, we subsequently ran additional validation studies with input signal yields up to twice 
Table 1: Summary of the systematic uncertainties expressed as a percent of the signal yield.

\begin{tabular}{|c|c|c|}
\hline & Source & Uncertainty on $N_{\text {sig }}(\%)$ \\
\hline$B^{0} \bar{B}^{0}$ counting & & 1.7 \\
\hline Tracking efficiency & & 0.2 \\
\hline Track multiplicity & & 3.4 \\
\hline Photon efficiency & & 4.0 \\
\hline Cluster time & & 0.7 \\
\hline$L_{\pi^{0}}$ and $L_{\eta}$ & & 2.8 \\
\hline Neural network & & 3.0 \\
\hline Fit uncertainty & & 9.9 \\
\hline Sum in quadrature & & 12.1 \\
\hline
\end{tabular}

the fit result. We observed a small fit bias, which we estimate to be $0.5 \pm 0.1$ events when the input signal equals the result of our fit. Consequently, we subtract this bias from our fit result, giving a corrected signal yield of $20.8_{-11.8}^{+12.8}$ events.

Systematic uncertainties affecting the calculation of the branching fraction include uncertainties on the number of $B^{0} \bar{B}^{0}$ events in the dataset, signal efficiency, and the signal yield from the fit. Table 1 summarizes all contributions, which are added in quadrature to give a total systematic uncertainty of 2.6 events on the signal yield.

The branching fraction is calculated from the measured signal yield using

$$
\mathscr{B}\left(B^{0} \rightarrow \gamma \gamma\right)=\frac{N_{\text {sig }}}{\varepsilon_{\text {sig }} \cdot 2 \cdot N_{B^{0} \bar{B}^{0}}},
$$

where $N_{\text {sig }}$ is the signal yield from the maximum likelihood fit, $\varepsilon_{\text {sig }}$ is the signal selection efficiency determined from simulated $B^{0} \rightarrow \gamma \gamma$ events, and $N_{B^{0} \bar{B}^{0}}$ is the number of neutral $B$ meson pairs in the on-resonance dataset. We calculate the branching fraction to be

$$
\mathscr{B}\left(B^{0} \rightarrow \gamma \gamma\right)=(1.7 \pm 1.1 \pm 0.2) \times 10^{-7},
$$

with a statistical significance of $1.8 \sigma$, where the first error is statistical and the second is systematic.

The upper limit at the $90 \% \mathrm{CL}$ is obtained by integrating the likelihood curve resulting from the ML fit from zero to the value of $N_{\text {sig }}$ which contains $90 \%$ of the area under the curve. To incorporate the systematic uncertainty into the determination of the upper limit, the likelihood curve is convolved with a Gaussian shape whose width is equal to the total systematic uncertainty of 2.6 events. This yields a value of $N_{\text {sig }}=39$ events corresponding to an upper limit of

$$
\mathscr{B}\left(B^{0} \rightarrow \gamma \gamma\right)<3.2 \times 10^{-7} \quad(90 \% \mathrm{CL}) .
$$

This limit is nearly a factor of two below the best previous upper limit and is consistent with the SM branching fraction.

2. $B^{+} \rightarrow K^{+} \tau^{+} \tau^{-}$

In the SM, the di-lepton mass dependence of the $B^{+} \rightarrow K^{+} \tau^{+} \tau^{-}$decay rate has a shape similar to that of $B^{+} \rightarrow K^{+} \mu^{+} \mu^{-}$in the kinematically allowed high di-lepton mass region, with a branch- 

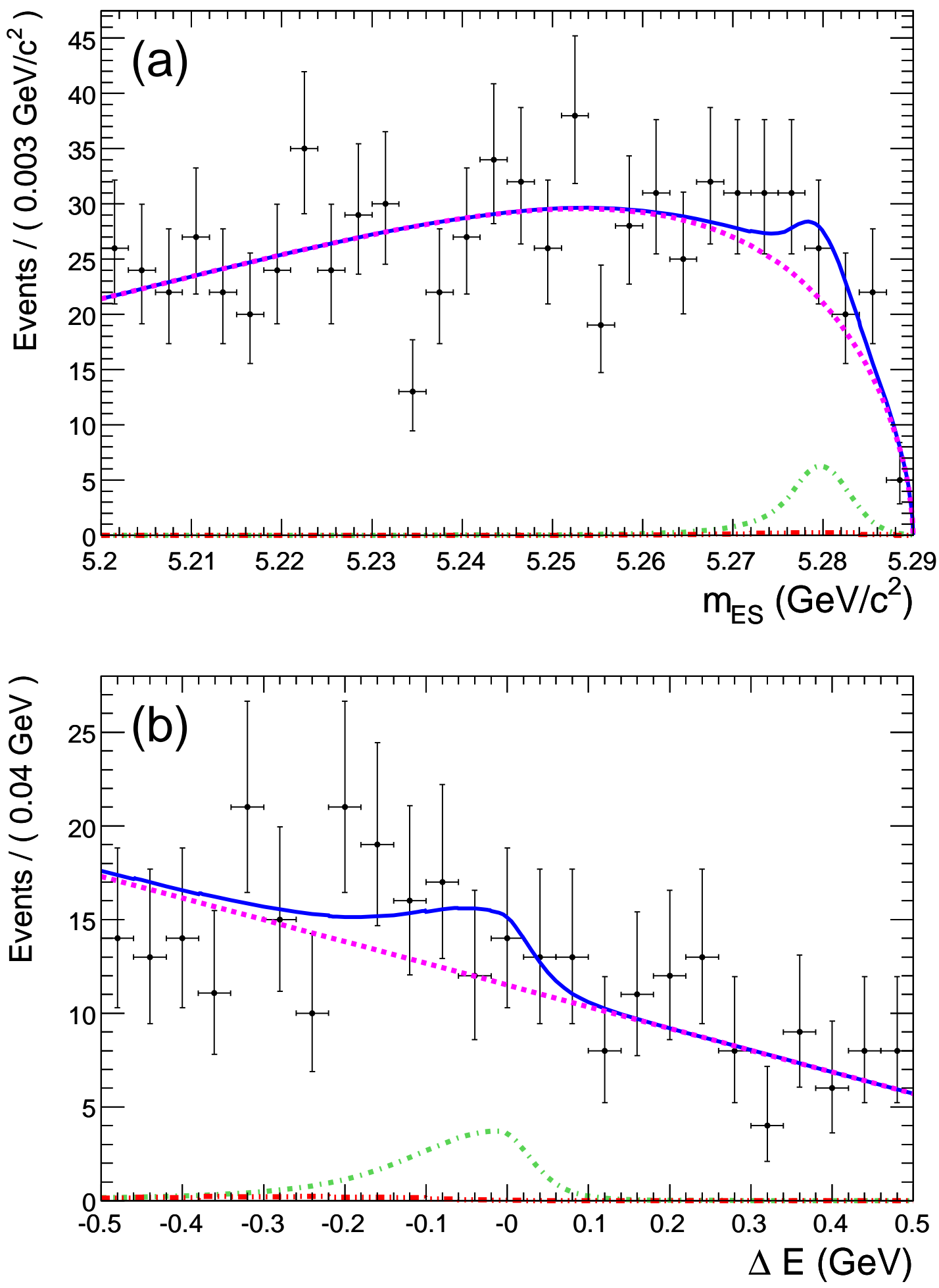

Figure 2: Projections of the ML fit onto $m_{\mathrm{ES}}$ and $\Delta E$. (a) The projection of the $m_{\mathrm{ES}}$ component when the range of $\Delta E$ has been restricted to $-0.30 \leq \Delta E \leq 0.13 \mathrm{GeV}$. (b) The $\Delta E$ projection of the fit when the range of $m_{\mathrm{ES}}$ has been restricted to $m_{\mathrm{ES}}>5.27 \mathrm{GeV} / c^{2}$. The points represent the on-resonance data. The solid curve represents the total PDF, the dashed curve is the continuum background component, the dot-dashed curve is the signal component, and the long-dashed curve is the peaking background component. With an expected yield of approximately one event, the peaking background component is nearly indistinguishable from the $x$-axis. 
ing fraction predicted to be $\sim 2 \times 10^{-7}$ in the SM, or $\sim 50-60 \%$ of the total inclusive rate [11]. Enhancements to the rate are predicted in many new physics models. In the next-to-minimal supersymmetric models (NMSSM), for example, the rate may be enhanced by the squared tau-to-muon mass ratio $\left(m_{\tau} / m_{\mu}\right)^{2} \sim 280$.

$B A B A R$ has performed the first search for $B^{+} \rightarrow K^{+} \tau^{+} \tau^{-}$using an integrated luminosity of $423 \mathrm{fb}^{-1}$, corresponding to $465 \mathrm{M} B \bar{B}$ events. Since signal final states contain 2-4 neutrinos, a recoiling ("tag") $B$ is reconstructed in many hadronic final states, $B^{-} \rightarrow D^{(*) 0,+} X$, where $X$ represents up to six hadrons $\left(\pi^{ \pm}, \pi^{0}, K^{ \pm}, K_{S}^{0}\right)$, in order to kinematically select an event sample enriched in $B$ events. Using $m_{E S}$ and $\Delta E$, tag decays are selected with an efficiency of $\sim 0.2 \%$, while the single-prong $\tau$ decays $\tau \rightarrow e v \bar{v}, \tau \rightarrow \mu \nu \bar{v}$ and $\tau \rightarrow \pi \nu$ are selected as signal modes. Thus, signal candidates are required to have exactly three charged particles of which one is an identified kaon with charge opposite to the $\operatorname{tag} B$ and $0.44<p_{K}<1.4 \mathrm{GeV} / \mathrm{c}$ in the $\Upsilon(4 S) \mathrm{CM}$ frame. The two remaining particles must have opposite charge, be kinematically consistent with the signal $\tau$ decays, have $p<1.59 \mathrm{GeV} / \mathrm{c}$ and a mass $M_{\text {pair }}<2.89 \mathrm{GeV} / c^{2}$. Further requirements are $q^{2}=\left(\vec{p}_{r(4 S)}-\vec{p}_{t a g}-\vec{p}_{K}\right)^{2} / c^{2}>14.23 \mathrm{GeV}^{2} / \mathrm{c}^{4}$, a missing energy (estimated as the difference between the $\Upsilon(4 S)$ energy and that of all observed particles in an event) of $1.39<E_{\text {miss }}<3.38 \mathrm{GeV}$, and neutral energy deposited in the electromagnetic calorimeter $E_{\text {extra }}<0.74 \mathrm{GeV}$. Continuum background is suppressed by $\left|\cos \theta_{T}\right|<0.8$, where $\theta_{T}$ is the opening angle between the thrust axis of the tag and that of the rest of the event. The largest remaining background originates from $B^{+} \rightarrow D^{0} X^{+}$, which is suppressed by combining the signal $K^{+}$with the $\tau$ daughter of opposite charge assigned the $\pi$ mass hypothesis and requiring a mass $M_{K \pi}>1.96 \mathrm{GeV} / \mathrm{c}^{2}$.

We observe 47 events with an expected background of $64.7 \pm 7.3$ events. Including systematic uncertainties, a branching fraction upper limit of $\mathscr{B}\left(B^{+} \rightarrow K^{+} \tau^{+} \tau^{-}\right)<3.3 \times 10^{-3}$ is set at the $90 \%$ confidence level (CL).

\section{References}

[1] S.W. Bosch and G. Buchalla, JHEP 0208:054 (2002).

[2] T. M. Aliev and E. O. Iltan, Phys. Rev. D 58, 095014 (1998).

[3] A. Gemintern, S. Bar-Shalom, and G. Eilam, Phys. Rev. D 70, 035008 (2004).

[4] S. Villa et al. (Belle Collaboration), Phys. Rev. D 73, 051107 (2006).

[5] J. Wicht et al. (Belle Collaboration), Phys. Rev. Lett. 100, 121801 (2008).

[6] B. Aubert et al. (BABAR Collaboration), Nucl. Inst. Meth. A 479, 1 (2002).

[7] K. Nakamura et al. (Particle Data Group), J. Phys. G 37, 075021 (2010).

[8] G.C. Fox and S. Wolfram, Phys. Rev. Lett.41, 1581 (1978).

[9] J. E. Gaiser et al., Phys. Rev. D 34, 711 (1986).

[10] H. Albrecht et al. (ARGUS Collaboration), Z. Phys. C 48, 543 (1990).

[11] J.L. Hewett, Phys. Rev. D53, 4964 (1995). 